\title{
On the motion of a neutrally buoyant ellipsoid in a three-dimensional Poiseuille flow
}

\author{
Tsorng-Whay Pan ${ }^{\mathrm{a}, *}$, Chien-Cheng Chang ${ }^{\mathrm{b}}$, Roland Glowinski ${ }^{\mathrm{a}}$ \\ ${ }^{a}$ University of Houston, Department of Mathematics, Houston, TX 77204, USA \\ ${ }^{\mathrm{b}}$ Academia Sinica, Research Center for Applied Sciences, and National Taiwan University, Institute of Applied Mechanics, Taipei, Taiwan, ROC
}

Received 20 February 2007; received in revised form 3 September 2007; accepted 4 September 2007

Available online 20 September 2007

This paper is dedicated to Professor Charles Peskin for his 60th birthday

\begin{abstract}
In previous articles, the authors introduced Lagrange multiplier based fictitious domain methods. Their goal in the present article is to apply a generalization of the above methods to simulate the motion of a neutrally buoyant ellipsoid in a three-dimensional Poiseuille flow and to investigate its rotational and orientational behavior via direct numerical simulations. We find distinctive states depending on the Reynolds number ranges and the shape of the ellipsoid.

(C) 2007 Elsevier B.V. All rights reserved.
\end{abstract}

Keywords: Solid-liquid flow; Neutrally buoyant ellipsoid; Poiseuille flow; Fictitious domain method; Finite element method

\section{Introduction}

The motion of particles in a channel is relevant to a variety of applications in many chemical engineering and biological processes, such as suspension process, sedimentation, blood flow, and flow cytometry. Understanding this motion has become more important with the recent advent of microfluidic devices used for many cell-based assays. The study of the motion of non-spherical particles in viscous fluids has a long history. Jeffery [16] solved the motion of a free ellipsoid in various types of unbounded shear flow under Stokes flow conditions. He concluded that the final state of a spheroid depended on its initial orientation and possesses the minimum energy dissipation. The experiments of Segré and Silberberg [32,33] have had a large influence on fluid mechanics studies of migration and lift of particles. They studied the migration of dilute suspensions of neutrally buoyant spheres in a tube flow. The particles migrate away from the wall and centerline and accumulate at about

\footnotetext{
* Corresponding author. Tel.: +1 713743 3448; fax: +1 7137433505 .

E-mail address: pan@math.uh.edu (T.-W. Pan).
}

0.6 of the tube radius from the centerline. Karnis et al. [17] verified the same phenomenon and observed, in contrary to Jeffery's theory, that the inertial effect migrates nonspherical particles to a final equilibrium distance in the tube at which the long axis of a rod-like particle rotates within the plane passing through the central axis of the tube and the mass center of the particle; but for a disk-like particle, it rotates with its short axis perpendicular to the plane passing through the central axis of the tube and the mass center of the disk. Comprehensive reviews of experimental and theoretical works have been given by Brenner [3], Cox and Mason [6], Feuillebois [10], Harper and Chang [15], Leal [18], and McLaughlin [20] among others.

Direct numerical simulations have been used for understanding particle motion in shear flows. Feng et al. [9] investigated the motion of neutrally buoyant and non-neutrally buoyant circular particles in plane Couette and Poiseuille flows using a finite element method and obtained qualitative agreement with the results of perturbation theories and of experiments. Ding and Aidun [8] has used a lattice Boltzmann method to study the effect of inertia on the dynamics of a solid particle (a disk, an ellipse, and an 
ellipsoid) suspended in shear flow. Qi and Luo [29,30] have used a lattice Boltzmann method to study the rotational and orientational behavior of a neutrally buoyant ellipsoidal particle in Couette flow and found different states depending on the Reynolds number ranges and the shape of particle. Pan and Glowinski have generalized the distributed Lagrange multiplier/fictitious domain method (DLM/ FD) for the numerical simulation of particulate flow (see $[11,13,14])$ to the case where the particles are neutrally buoyant in [22] for two-dimensional flows and confirmed via simulations that the phenomenon of collisions between particles is one of the key factors driving particles to the central region of the plane Poiseuille flow. Concerning three-dimensional computational results, Yang et al. [34] have recently studied the migration of a neutrally buoyant ball in a tube Poiseuille flow by using an arbitrary Lagrangian-Eulerian moving mesh technique. Pan and Glowinski [23] have extended the DLM/FD method to simulate the migration of a neutrally buoyant ball in a three-dimensional tube Poiseuille flow. Their results are in good agreement with those obtained in [34]. Yu et al. [35] have also investigated extensively the motion of a spherical particle in a tube including non-neutrally and almost neutrally buoyant cases via a finite-difference-based DLM/FC method. In this article, we have extended the DLM/FD method in [23] to the situation of non-spherical particles and performed simulations of the motion of a neutrally buoyant ellipsoid in a tube Poiseuille flow. We have investigated the migration and rotational behavior of a neutrally buoyant ellipsoid at Reynolds numbers up to 80 and found its rotation exhibits distinctive states depending on the Reynolds number ranges and the shape of particle. The content of this article is as follows: In Section 2, we discuss a fictitious domain formulation of the model problem concerning the case of a neutrally buoyant particle of general shape moving freely in a three-dimensional Poiseuille flow; then in Section 3 we discuss briefly the time and space discretization issues, and in Section 4 we present and comment the results of numerical experiments involving neutrally buoyant prolate and oblate ellipsoids.

\section{A fictitious domain formulation of the model problem}

All the fluid-solid interactions to be considered in this article concern the flow of fluid-solid particle mixtures in a cylindrical tube (denoted by $\mathbf{T}$ in the sequel) with a circular cross-section. In order to take a full advantage of the fictitious domain approach we will embed $\mathbf{T}$ in a cylindrical tube (denoted by $\Omega$ ) with a square cross-section whose edge length is equal to the diameter of the $\mathbf{T}$ cross-section. We will start our discussion with a one particle situation. Therefore, let $\Omega \subset \mathbb{R}^{3}$ be a rectangular parallelepiped. We suppose that $\Omega$ is filled with a Newtonian incompressible viscous fluid (of density $\rho_{f}$ and viscosity $\mu_{f}$ ) and that it contains a moving neutrally buoyant rigid particle $B$ centered at $\mathbf{G}=\left\{G_{1}, G_{2}, G_{3}\right\}^{\mathrm{t}}$ of density $\rho_{f}$, as shown in Fig. 1, which shows also the inclusion in $\Omega$ of the cylinder $\mathbf{T}$ mentioned

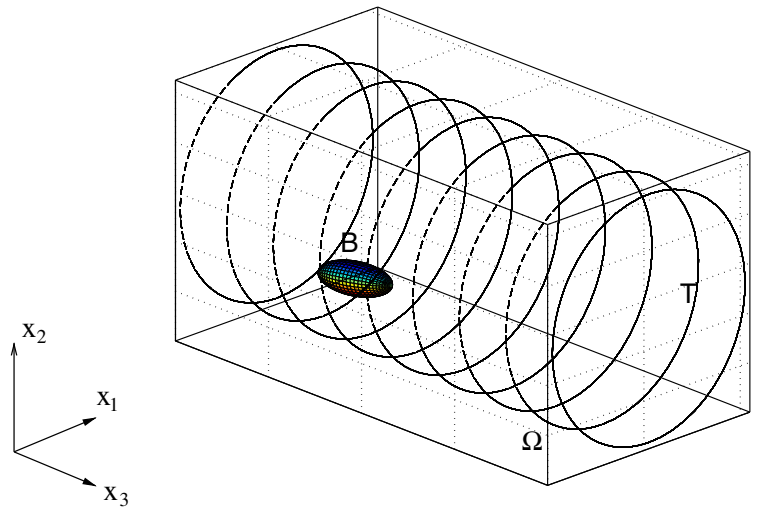

Fig. 1. An example of three-dimensional flow region with one rigid body.

above; we suppose that the central axis of both cylinders is parallel to the $x_{3}$-axis. The flow is modeled by the Navier-Stokes equations while the particle motion is described by the Euler-Newton's equations. We introduce (with $\mathrm{d} \mathbf{x}=\mathrm{d} x_{1} \mathrm{~d} x_{2} \mathrm{~d} x_{3}$ ) the following functional spaces:

$$
\begin{aligned}
& W_{0, P}=\left\{\mathbf{v} \mid \mathbf{v} \in\left(H^{1}(\Omega)\right)^{3},\right. \\
& \mathbf{v}=\mathbf{0} \text { on the top, bottom, front, }
\end{aligned}
$$

and back of $\Omega$ and $\mathbf{v}$ is periodic in the $x_{3}$ direction\},

$$
\begin{aligned}
& L_{0}^{2}=\left\{q \mid q \in L^{2}(\Omega), \int_{\Omega} q \mathrm{~d} \mathbf{x}=0\right\}, \\
& \Lambda_{0}(t)=\left\{\boldsymbol{\mu} \mid \boldsymbol{\mu} \in\left(H^{1}(B(t))\right)^{3},<\boldsymbol{\mu}, \mathbf{e}_{i}>_{B(t)}=0,\right. \\
& \left.\quad<\boldsymbol{\mu}, \mathbf{e}_{i} \times \overrightarrow{\mathbf{G}}>_{B(t)}=0, \quad i=1,2,3\right\},
\end{aligned}
$$

$\Lambda_{T}=\left\{\boldsymbol{\mu} \mid \boldsymbol{\mu} \in\left(H^{1}(\Omega \backslash \overline{\mathbf{T}})\right)^{3}, \boldsymbol{\mu}\right.$ is periodic in the $x_{3}$ direction $\}$

where $\mathbf{e}_{1}=\{1,0,0\}^{\mathrm{t}}, \quad \mathbf{e}_{2}=\{0,1,0\}^{\mathrm{t}}, \quad \mathbf{e}_{3}=\{0,0,1\}^{\mathrm{t}}$, and where $\langle\cdot, \cdot\rangle_{B(t)}$ (resp., $\langle\cdot, \cdot\rangle_{T}$ ) is an inner product on $\Lambda_{0}(t)$ (resp., $\Lambda_{T}$ ) (see [14] (Section 5) and [11] (Chapter 8) for further information on the choice of $\left.\langle\cdot \cdot\rangle_{B(t)}\right)$. Above, and from now on, periodicity in the $x_{3}$ direction means periodicity of period $L, L$ being the common length of the truncated cylinders $\Omega$ and $\mathbf{T}$. Then, the distributed Lagrange multiplier based fictitious domain formulation for the flow around a freely moving neutrally buoyant particle of general shape inside a cylindrical tube reads as follows (see $[24,25]$ for a detailed discussion of the non-neutrally buoyant case):

$$
\begin{aligned}
& \text { For a.e. } t>0 \text {, find } \mathbf{u}(t) \in W_{0, P}, p(t) \in L_{0}^{2}, \mathbf{V}_{\mathbf{G}}(t) \\
& \qquad \begin{array}{l}
\in \mathbb{R}^{3}, \mathbf{G}(t) \in \mathbb{R}^{3}, \boldsymbol{\omega}(t) \in \mathbb{R}^{3}, \boldsymbol{\lambda}(t) \in \Lambda_{0}(t), \boldsymbol{\lambda}_{T} \\
\in \Lambda_{T} \text { such that }
\end{array} \\
& \qquad \begin{array}{c}
\rho_{f} \int_{\Omega}\left[\frac{\partial \mathbf{u}}{\partial t}+(\mathbf{u} \cdot \boldsymbol{\nabla}) \mathbf{u}\right] \cdot \mathbf{v} \mathrm{d} \mathbf{x}+2 \mu_{f} \int_{\Omega} \mathbf{D}(\mathbf{u}): \mathbf{D}(\mathbf{v}) \mathrm{d} \mathbf{x} \\
-\int_{\Omega} p \boldsymbol{\nabla} \cdot \mathbf{v} \mathrm{d} \mathbf{x}-<\boldsymbol{\lambda}, \mathbf{v}>_{B(t)}-<\lambda_{T}, \mathbf{v}>_{T} \\
=\rho_{f} \int_{\Omega} \mathbf{g} \cdot \mathbf{v} \mathrm{d} \mathbf{x}+\int_{\Omega} \mathbf{F} \cdot \mathbf{v} \mathrm{d} \mathbf{x}, \quad \forall \mathbf{v} \in W_{0, P},
\end{array}
\end{aligned}
$$


$\int_{\Omega} q \boldsymbol{\nabla} \cdot \mathbf{u}(t) \mathrm{d} \mathbf{x}=0, \quad \forall q \in L^{2}(\Omega)$,

$\langle\boldsymbol{\mu}, \quad \mathbf{u}(t)\rangle_{B(t)}=0, \quad \forall \boldsymbol{\mu} \in \Lambda_{0}(t)$,

$\left\langle\boldsymbol{\mu}_{T}, \quad \mathbf{u}(t)\right\rangle_{T}=0, \quad \forall \boldsymbol{\mu}_{T} \in \Lambda_{T}$,

$\frac{\mathrm{d} \mathbf{G}}{\mathrm{d} t}=\mathbf{V}_{\mathbf{G}}$,

$\frac{\mathrm{d} \mathbf{x}_{i}}{\mathrm{~d} t}=\mathbf{V}_{\mathbf{G}}+\omega \times \overrightarrow{\mathbf{G}} \mathbf{x}_{i}, \quad i=1,2$,

$\mathbf{V}_{\mathbf{G}}(0)=\mathbf{V}_{\mathbf{G}}^{0}, \quad \omega(0)=\omega^{0}$,

$\mathbf{G}(0)=\mathbf{G}^{0}=\left\{G_{1}^{0}, G_{2}^{0}, G_{3}^{0}\right\}^{\mathrm{t}}, \quad \mathbf{x}_{i}(0)=\mathbf{x}_{i, 0}, \quad i=1,2$,

$\mathbf{u}(\mathbf{x}, 0)=\overline{\mathbf{u}}_{0}(\mathbf{x})= \begin{cases}\mathbf{u}_{0}(\mathbf{x}), & \forall \mathbf{x} \in \Omega \backslash \overline{B(0)} \\ \mathbf{V}_{\mathbf{G}}^{0}+\boldsymbol{\omega}^{0} \times \mathbf{G}^{0} \mathbf{x}, & \forall \mathbf{x} \in \overline{B(0)} .\end{cases}$

In (1)-(8) $\mathbf{u}$ and $p$ denote velocity and pressure, respectively, $\lambda$ is a Lagrange multiplier associated with relation (3) (from (3) the fluid has a rigid body motion in the region occupied by $B(t)), \lambda_{T}$ is a Lagrange multiplier associated with relation (4) (from (4), the fluid velocity is $\mathbf{0}$ in $\bar{\Omega} \backslash \mathbf{T}), \mathbf{D}(\mathbf{v})=\frac{1}{2}\left(\nabla \mathbf{v}+(\nabla \mathbf{v})^{t}\right), \mathbf{g}$ denotes gravity, $\mathbf{F}$ is an imposed pressure gradient pointing in the $x_{3}$-direction inside the cylinder $\mathbf{T}, \mathbf{V}_{\mathbf{G}}$ is the translation velocity of the particle $B$, and $\omega$ is the angular velocity of $B$. We suppose that the no-slip condition holds on $\partial B$. We also use, if necessary, the notation $\phi(t)$ for the function $\mathbf{x} \rightarrow \phi(\mathbf{x}, t)$.

Remark 1. The hydrodynamical forces and torque imposed on the rigid body by the fluid are built in (1)-(8) implicitly (see $[13,14]$ for details), thus we do not need to compute them explicitly in the simulation. Since in (1)-(8) the flow field is defined on the entire domain $\Omega$, it can be computed with a simple structured grid. The fictitious domain methods that we advocate have some common features with the immersed boundary method of Ch. Peskin (see, e.g., Refs. [26-28]) but also some significant differences in the sense that we take systematically advantage of distributed Lagrange multipliers to force the rigid body motion inside the particle.

Remark 2. In (3), the rigid body motion in the region occupied by the particle is enforced via Lagrange multipliers $\lambda$. To recover the translation velocity $\mathbf{V}_{\mathbf{G}}(t)$ and the angular velocity $\boldsymbol{\omega}(t)$ from $\mathbf{u}(t)$ satisfying (3), we solve the following equations

$$
\left\{\begin{array}{l}
\left\langle\mathbf{e}_{i}, \mathbf{u}(t)-\mathbf{V}_{\mathbf{G}}(t)-\boldsymbol{\omega}(t) \times \overrightarrow{\mathbf{G}} \mathbf{x}\right\rangle_{B(t)}=0, \\
\quad \text { for } i=1,2,3, \\
\left\langle\mathbf{e}_{i} \times \overrightarrow{\mathbf{G x}}, \mathbf{u}(t)-\mathbf{V}_{\mathbf{G}}(t)-\boldsymbol{\omega}(t) \times \overrightarrow{\mathbf{G x}}\right\rangle_{B(t)}=0, \\
\quad \text { for } i=1,2,3 .
\end{array}\right.
$$

Remark 3. In (6), we have to track the motion of two extra points attached to any particle of general shape so that we can determine the region occupied by the particle via its center of mass, the translation velocity of the center of mass and the angular velocity of the particle. In practice, we shall track two orthogonal normalized vectors rigidly attached to the body $B$ from the center of mass $\mathbf{G}$.

Remark 4. In (1), $2 \int_{\Omega} \mathbf{D}(\mathbf{u}): \mathbf{D}(\mathbf{v}) \mathrm{d} \mathbf{x}$ can be replaced by $\int_{\Omega} \nabla \mathbf{u}: \nabla \mathbf{v} \mathrm{d} \mathbf{x}$ since $\mathbf{u}$ is divergence free and in $W_{0, P}$. This change can make the computation simpler and faster. Also the gravity $\mathbf{g}$ in (1) can be absorbed into the pressure term.

\section{Space approximation and time discretization}

Concerning the finite element based space approximation of $\{\mathbf{u}, p\}$ in problem (1)-(8), we will use the BercovierPironneau $P_{1}$-iso- $P_{2}$ finite element approximation (as in Bristeau et al. [4]; see also [11] (Chapter 5)). More precisely, with $h$ a space discretization step we introduce a uniform "tetrahedrization" $\mathscr{T}_{h}$ of $\bar{\Omega}$ and a twice coarser "tetrahedrization" $\mathscr{T}_{2 h}$. We approximate then $W_{0, P}, L^{2}(\Omega)$ and $L_{0}^{2}$ by the following finite dimensional spaces

$W_{0, h}=\left\{\mathbf{v}_{h}\left|\mathbf{v}_{h} \in\left(C^{0}(\bar{\Omega})\right)^{3}, \mathbf{v}_{h}\right|_{T} \in\left(P_{1}\right)^{3}, \forall T \in \mathscr{T}_{h}\right.$,

$\mathbf{v}_{h}=\mathbf{0}$ on the top, bottom, front, and back of $\Omega$

and $\mathbf{v}$ is periodic at $\Gamma$ in the $x_{3}$ direction,

$L_{h}^{2}=\left\{q_{h}\left|q_{h} \in C^{0}(\bar{\Omega}), q_{h}\right|_{T} \in P_{1}, \quad \forall T \in \mathscr{T}_{2 h}\right\}$,

$L_{0, h}^{2}=\left\{q_{h} \mid q_{h} \in L_{h}^{2}, \int_{\Omega} q_{h} \mathrm{~d} \mathbf{x}=0, q_{h}\right.$ is periodic at $\Gamma$

$$
\text { in the } \left.x_{3} \text { direction }\right\} \text {, }
$$

respectively; in (10)-(12), $P_{1}$ is the space of the polynomials in three variables of degree $\leqslant 1$. A finite dimensional space approximating $\Lambda_{0}(t)$ is as follows: let $\left\{\boldsymbol{\xi}_{i}\right\}_{i=1}^{N}$ be a set of points from $\overline{B(t)}$ which cover $\overline{B(t)}$ (uniformly, for example); we define then

$\Lambda_{h}(t)=\left\{\boldsymbol{\mu}_{h} \mid \boldsymbol{\mu}_{h}=\sum_{i=1}^{N} \boldsymbol{\mu}_{i} \delta\left(\mathbf{x}-\boldsymbol{\xi}_{i}\right), \boldsymbol{\mu}_{i} \in \mathbb{R}^{3}, \forall i=1, \ldots, N\right\}$,

where $\delta(\cdot)$ is the Dirac measure at $\mathbf{x}=\mathbf{0}$. Then we shall use $\langle\cdot, \cdot\rangle_{B_{h}(t)}$ defined by

$\left\langle\boldsymbol{\mu}_{h}, \mathbf{v}_{h}\right\rangle_{B_{h}(t)}=\sum_{i=1}^{N} \boldsymbol{\mu}_{i} \cdot \mathbf{v}_{h}\left(\boldsymbol{\xi}_{i}\right), \quad \forall \boldsymbol{\mu}_{h} \in \Lambda_{h}(t), \quad \mathbf{v}_{h} \in W_{0, h}$.

Then we approximate $\Lambda_{0}(t)$ by

$$
\begin{gathered}
\Lambda_{0, h}(t)=\left\{\boldsymbol{\mu} \mid \boldsymbol{\mu} \in \Lambda_{h}(t),\left\langle\boldsymbol{\mu}, \mathbf{e}_{i}\right\rangle_{B_{h}(t)}=0,\right. \\
\left.\left\langle\boldsymbol{\mu}, \mathbf{e}_{i} \times \overrightarrow{\mathbf{G}} \mathbf{x}\right\rangle_{B_{h}(t)}=0, i=1,2,3\right\} .
\end{gathered}
$$

A typical choice of points for defining (13) is to take the grid points of the velocity mesh internal to the particle $B$ 


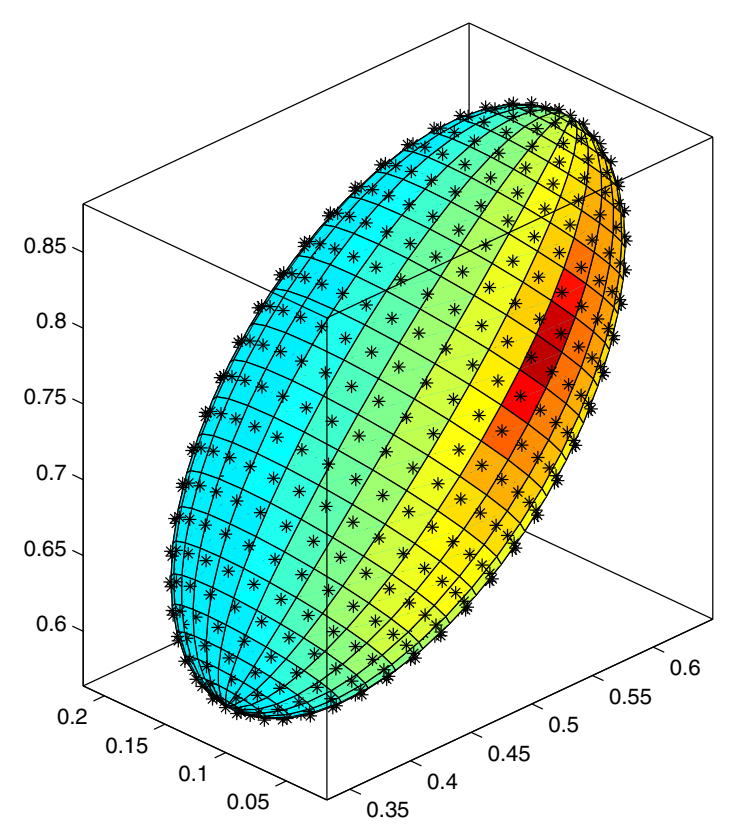

Fig. 2. An example of selected points on the boundary of the particle.

and whose distance to the boundary of $B$ is greater than, e.g. $h / 2$, and to complete with selected points from the boundary of $B(t)$ (see, e.g., Fig. 2, for an example of selected points on the surface of $B(t)$ ). As we did for $\Lambda_{h}(t)$ and $\langle\cdot, \cdot\rangle_{B_{h}(t)}$, we define the finite dimensional space $\Lambda_{T, h}$ and the inner product $\langle\cdot, \cdot\rangle_{T_{h}}$ via a set of points of the velocity mesh internal to the region $\Omega \backslash \overline{\mathbf{T}}$ and whose distance to the surface of $\mathbf{T}$ is greater than, e.g. $h$, and a set of the points chosen from the surface of the cylinder $\mathbf{T}$.

Remark 5. In order to facilitate the construction of the finite dimensional space $\Lambda_{T, h}$ we slightly increased the size of the cross-section of $\Omega$, replacing thus this last cylinder by $\Omega_{h}$. With this approach it is easier to select the collocation points used to force the condition $\mathbf{u}=\mathbf{0}$ outside $\mathbf{T}$ (see Section 4 for more details).

Remark 6. The inner product like bracket $\langle\cdot, \cdot\rangle_{B_{h}(t)}$ in (14) makes little sense for the continuous problem, but it is meaningful for the discrete problem; it amounts to forcing the rigid body motion of $B(t)$ via a collocation method. A similar technique has been used to enforce Dirichlet boundary conditions by Bertrand et al. [2].

Using the above finite dimensional spaces leads to the following approximation of problem (1)-(8) (where for notational simplicity, we still denote by $\Omega$ the domain $\Omega_{h}$ introduced in Remark 5):

For a.e. $t>0$, find $\mathbf{u}_{h}(t) \in W_{0, h}, p(t) \in L_{0, h}^{2}$,

$\mathbf{V}_{\mathbf{G}}(t) \in \mathbb{R}^{3}, \quad \mathbf{G}(t) \in \mathbb{R}^{3}, \quad \omega(t) \in \mathbb{R}^{3}, \quad \lambda_{h}(t) \in \Lambda_{0, h}(t)$,

$\lambda_{T_{h}} \in \Lambda_{T, h}$ such that

$\left\{\begin{array}{l}\rho_{f} \int_{\Omega}\left[\frac{\partial \mathbf{u}_{h}}{\partial t}+\left(\mathbf{u}_{h} \cdot \nabla\right) \mathbf{u}_{h}\right] \cdot \mathbf{v} \mathrm{d} \mathbf{x}+\mu_{f} \int_{\Omega} \nabla \mathbf{u}_{h}: \nabla \mathbf{v} \mathrm{d} \mathbf{x} \\ -\int_{\Omega} p_{h} \boldsymbol{\nabla} \cdot \mathbf{v} \mathrm{d} \mathbf{x}-\left\langle\boldsymbol{\lambda}_{h}, \mathbf{v}\right\rangle_{B_{h}(t)}-\left\langle\boldsymbol{\lambda}_{T_{h}}, \mathbf{v}\right\rangle_{T_{h}} \\ \quad=\int_{\Omega} \mathbf{F} \cdot \mathbf{v} \mathrm{d} \mathbf{x}, \quad \forall \mathbf{v} \in W_{0, h},\end{array}\right.$
$\int_{\Omega} q \nabla \cdot \mathbf{u}_{h}(t) \mathrm{d} \mathbf{x}=0, \quad \forall q \in L_{h}^{2}$,

$\left\langle\boldsymbol{\mu}, \mathbf{u}_{h}(t)\right\rangle_{B_{h}(t)}=0, \quad \forall \boldsymbol{\mu} \in \Lambda_{0, h}(t)$,

$\left\langle\boldsymbol{\mu}_{T}, \mathbf{u}_{h}(t)\right\rangle_{T_{h}}=0, \quad \forall \boldsymbol{\mu}_{T} \in \Lambda_{T, h}$,

$\frac{\mathrm{d} \mathbf{G}}{\mathrm{d} t}=\mathbf{V}_{\mathbf{G}}$,

$\frac{\mathrm{d} \mathbf{x}_{i}}{\mathrm{~d} t}=\mathbf{V}_{\mathbf{G}}+\omega \times \overrightarrow{\mathbf{G}} \mathbf{x}_{i}, \quad i=1,2$,

$\mathbf{V}_{\mathbf{G}}(0)=\mathbf{V}_{\mathbf{G}}^{0}, \quad \omega(0)=\omega^{0}, \quad \mathbf{G}(0)=\mathbf{G}^{0}$

$=\left\{G_{1}^{0}, G_{2}^{0}, G_{3}^{0}\right\}^{\mathrm{t}}, \quad \mathbf{x}_{i}(0)=\mathbf{x}_{i, 0}, \quad i=1,2$,

$\mathbf{u}_{h}(\mathbf{x}, 0)=\overline{\mathbf{u}}_{0, h}(\mathbf{x})\left(\right.$ with $\left.\nabla \cdot \overline{\mathbf{u}}_{0, h}=0\right)$.

Applying a first order operator splitting scheme à la Marchuk-Yanenko [19] (also see $[11,13,14]$ ) to discretize Eqs. (16)-(23) in time, we obtain (after dropping some of the subscripts $h$ ):

$\mathbf{u}^{0}=\overline{\mathbf{u}}_{0, h}, \mathbf{V}_{\mathbf{G}}^{0}, \boldsymbol{\omega}^{0}, \mathbf{G}^{0}, \quad \mathbf{x}_{1}^{0}=\mathbf{x}_{1,0}, \quad \mathbf{x}_{2}^{0}=\mathbf{x}_{2,0}$ given;

for $n \geqslant 0$, knowing $\mathbf{u}^{n}, \mathbf{V}_{\mathbf{G}}{ }^{n}, \boldsymbol{\omega}^{n}, \mathbf{G}^{n}, \mathbf{x}_{1}^{n}$ and $\mathbf{x}_{2}^{n}$, compute $\mathbf{u}^{n+\frac{1}{6}}$ and $p^{n+\frac{1}{6}}$ via the solution of

$\left\{\begin{array}{l}\rho_{f} \int_{\Omega} \frac{\mathbf{u}^{n+\frac{1}{6}}-\mathbf{u}^{n}}{\Delta t} \cdot \mathbf{v} \mathrm{d} \mathbf{x}-\int_{\Omega} p^{n+\frac{1}{6}} \nabla \cdot \mathbf{v} \mathrm{d} \mathbf{x}=0, \\ \quad \forall \mathbf{v} \in W_{0, h}, \\ \int_{\Omega} q \nabla \cdot \mathbf{u}^{n+\frac{1}{6}} \mathrm{~d} \mathbf{x}=0, \\ \quad \forall q \in L_{h}^{2} ; \mathbf{u}^{n+\frac{1}{6}} \in W_{0, h}, p^{n+\frac{1}{6}} \in L_{0, h}^{2} .\end{array}\right.$

Then compute $\mathbf{u}^{n+\frac{2}{6}}$ via the solution of

$\left\{\begin{array}{l}\int_{\Omega} \frac{\partial \mathbf{u}}{\partial t} \cdot \mathbf{v} \mathrm{d} \mathbf{x}+\int_{\Omega}\left(\mathbf{u}^{n+\frac{1}{6}} \cdot \nabla\right) \mathbf{u} \cdot \mathbf{v} \mathrm{d} \mathbf{x}=0, \\ \quad \forall \mathbf{v} \in W_{0, h}, \text { a.e. on }\left(t^{n}, t^{n+1}\right), \\ \mathbf{u}\left(t^{n}\right)=\mathbf{u}^{n+\frac{1}{6}} ; \quad \mathbf{u}(t) \in W_{0, h},\end{array}\right.$

$\mathbf{u}^{n+\frac{2}{6}}=\mathbf{u}\left(t^{n+1}\right)$.

Next, compute $\mathbf{u}^{n+\frac{3}{6}}$ and $\lambda_{T_{h}}^{n+\frac{3}{6}}$ via the solution of

$\left\{\begin{array}{l}\rho_{f} \int_{\Omega} \frac{\mathbf{u}^{n+\frac{3}{6}}-\mathbf{u}^{n+\frac{2}{6}}}{\Delta t} \cdot \mathbf{v} \mathrm{d} \mathbf{x}+\alpha \mu_{f} \int_{\Omega} \nabla \mathbf{u}^{n+\frac{3}{6}} \cdot \nabla \mathbf{v} \mathrm{d} \mathbf{x} \\ \quad-\left\langle\lambda_{T_{h}}^{n+\frac{3}{6}}, \mathbf{v}\right\rangle_{T_{h}}=\int_{\Omega} \mathbf{F}^{n+1} \cdot \mathbf{v} \mathrm{d} \mathbf{x}, \quad \forall \mathbf{v} \in W_{0, h}, \\ \left\langle\boldsymbol{\mu}_{T}, \mathbf{u}^{n+\frac{3}{6}}\right\rangle_{T_{h}}=0, \\ \forall \boldsymbol{\mu}_{T} \in \Lambda_{T, h} ; \mathbf{u}^{n+\frac{3}{6}} \in W_{0, h}, \lambda_{T_{h}}^{n+\frac{3}{6}} \in \Lambda_{T, h} .\end{array}\right.$

Now predict the motion of the center of mass and the angular velocity of the particle via

$\frac{\mathrm{d} \mathbf{G}}{\mathrm{d} t}=\mathbf{V}_{\mathbf{G}}(t) / 2$,

$M_{p} \frac{\mathrm{d} \mathbf{V}_{\mathbf{G}}}{\mathrm{d} t}=\mathbf{F}_{r, \|} / 2$,

$\frac{\mathrm{d}\left(\mathbf{I}_{p} \boldsymbol{\omega}\right)}{\mathrm{d} t}=\overrightarrow{\mathbf{G x}_{r}} \times \mathbf{F}_{r, \perp} / 2$,

$\frac{\mathrm{d} \mathbf{x}_{i}}{\mathrm{~d} t}=\mathbf{V}_{\mathbf{G}}(t)+\boldsymbol{\omega}(t) \times \overrightarrow{\mathbf{G}(t)} \mathbf{x}_{i}, \quad$ for $i=1,2$, 


$$
\begin{gathered}
\mathbf{G}\left(t^{n}\right)=\mathbf{G}^{n}, \mathbf{V}_{\mathbf{G}}\left(t^{n}\right)=\mathbf{V}_{\mathbf{G}}^{n},\left(\mathbf{I}_{p} \boldsymbol{\omega}\right)\left(t^{n}\right)=\left(\mathbf{I}_{p} \boldsymbol{\omega}\right)^{n}, \\
\mathbf{x}_{1}\left(t^{n}\right)=\mathbf{x}_{1}^{n}, \mathbf{x}_{2}\left(t^{n}\right)=\mathbf{x}_{2}^{n},
\end{gathered}
$$

for $t^{n}<t<t^{n+1}$. Then set $\mathbf{G}^{n+4 / 6}=\mathbf{G}\left(t^{n+1}\right), \quad \mathbf{V}_{\mathbf{G}}{ }^{n+4 / 6}=$ $\mathbf{V}_{\mathbf{G}}\left(t^{n+1}\right), \quad\left(\mathbf{I}_{p} \boldsymbol{\omega}\right)^{n+4 / 6}=\left(\mathbf{I}_{p} \boldsymbol{\omega}\right)\left(t^{n+1}\right), \quad \mathbf{x}_{1}^{n+4 / 6}=\mathbf{x}_{1}\left(t^{n+1}\right), \quad$ and $\mathbf{x}_{2}^{n+4 / 6}=\mathbf{x}_{2}\left(t^{n+1}\right)$.

Using $\mathbf{G}^{n+4 / 6}, \mathbf{x}_{1}^{n+4 / 6}$ and $\mathbf{x}_{2}^{n+4 / 6}$ obtained at the above step, we enforce the rigid body motion in the region $B_{h}^{n+4 / 6}$ occupied by the particle by computing $\mathbf{u}^{n+\frac{5}{6}}$ and $\lambda^{n+\frac{5}{6}}$ via the solution of

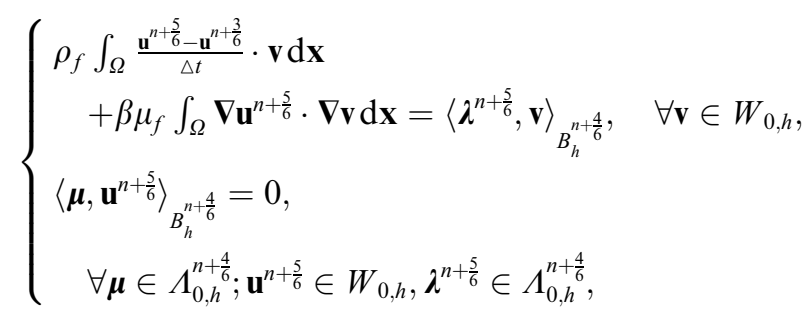

and then solve for $\mathbf{V}_{\mathbf{G}}^{n+\frac{5}{6}}$ and $\omega^{n+\frac{5}{6}}$ from $\mathbf{u}^{n+\frac{5}{6}}$

$$
\left\{\begin{array}{l}
\left\langle\mathbf{e}_{i}, \mathbf{u}^{n+\frac{5}{6}}-\mathbf{V}_{\mathbf{G}}^{n+\frac{5}{6}}-\boldsymbol{\omega}^{n+\frac{5}{6}} \times \mathbf{G}^{n+\frac{4}{6}} \mathbf{X}>_{B_{h}^{n+\frac{4}{6}}}=0,\right. \\
\quad \text { for } i=1,2,3, \\
\left\langle\mathbf{e}_{i} \times \mathbf{G}^{n+\frac{4}{6}} \mathbf{X}, \mathbf{u}^{n+\frac{5}{6}}-\mathbf{V}_{\mathbf{G}}^{n+\frac{5}{6}}-\boldsymbol{\omega}^{n+\frac{5}{6}}\right. \\
\left.\quad \times \mathbf{G}^{n+\frac{4}{6}} \mathbf{X}\right\rangle_{B_{h}^{n+\frac{4}{6}}}=0, \quad \text { for } i=1,2,3 .
\end{array}\right.
$$

Correct the motion of the center of mass and the angular velocity of the particle via

$$
\begin{aligned}
& \frac{\mathrm{d} \mathbf{G}}{\mathrm{d} t}=\mathbf{V}_{\mathbf{G}}(t) / 2 \\
& M_{p} \frac{\mathrm{d} \mathbf{V}_{\mathbf{G}}}{\mathrm{d} t}=\mathbf{F}_{r, \|} / 2 \\
& \frac{\mathrm{d}\left(\mathbf{I}_{p} \boldsymbol{\omega}\right)}{\mathrm{d} t}=\overrightarrow{\mathbf{G}}_{r} \times \mathbf{F}_{r, \perp} / 2 \\
& \frac{\mathrm{d} \mathbf{x}_{i}}{\mathrm{~d} t}=\mathbf{V}_{\mathbf{G}}(t)+\boldsymbol{\omega}(t) \times \mathbf{G}(\vec{t}) \mathbf{x}_{i}, \quad \text { for } i=1,2 \\
& \mathbf{G}\left(t^{n}\right)=\mathbf{G}^{n+4 / 6}, \quad \mathbf{V}_{\mathbf{G}}\left(t^{n}\right)=\mathbf{V}_{\mathbf{G}}^{n+5 / 6} \\
& \left(\mathbf{I}_{p} \boldsymbol{\omega}\right)\left(t^{n}\right)=\left(\mathbf{I}_{p} \boldsymbol{\omega}\right)^{n+5 / 6}, \quad \mathbf{x}_{1}\left(t^{n}\right)=\mathbf{x}_{1}^{n+4 / 6}, \quad \mathbf{x}_{2}\left(t^{n}\right)=\mathbf{x}_{2}^{n+4 / 6}
\end{aligned}
$$

for $t^{n}<t<t^{n+1}$. Then set $\mathbf{G}^{n+1}=\mathbf{G}\left(t^{n+1}\right), \quad \mathbf{V}_{\mathbf{G}}{ }^{n+1}=$ $\mathbf{V}_{\mathbf{G}}\left(t^{n+1}\right), \quad\left(\mathbf{I}_{p} \boldsymbol{\omega}\right)^{n+1}=\left(\mathbf{I}_{p} \boldsymbol{\omega}\right)\left(t^{n+1}\right), \quad \mathbf{x}_{1}^{n+1}=\mathbf{x}_{1}\left(t^{n+1}\right), \quad \mathbf{x}_{2}^{n+1}=$ $\mathbf{x}_{2}\left(t^{n+1}\right)$ and $\mathbf{u}^{n+1}=\mathbf{u}^{n+\frac{5}{6}}$.

In the above algorithm (24)-(40), we have $t^{n+s}=(n+s) \triangle t, \Lambda_{0, h}^{n+s}=\Lambda_{0, h}\left(t^{n+s}\right), \mathbf{F}_{r}$ is a short range repulsion force which prevents the particle/particle and particle/ wall penetration (see, e.g., $[13,14,25]$ ), and $\mathbf{x}_{r}$ is the point on which a torque associated with $\mathbf{F}_{r}$ applies to $B$. $\mathbf{F}_{r, \|}$ is the projection of $\mathbf{F}_{r}$ on $\mathbf{G} \mathbf{x}_{r}$ and $\mathbf{F}_{r, \perp}\left(=\mathbf{F}_{r}-\mathbf{F}_{r, \|}\right)$ is the one perpendicular to $\mathbf{G} \mathbf{x}_{r}$. Finally, $\alpha$ and $\beta$ verify $\alpha+\beta=1$; we have chosen $\alpha=1$ and $\beta=0$ for the numerical simulations to be discussed in Section 4 .
3.1. Solutions of the subproblems (25), (26), (28), (34), and (29)-(33)

After decoupling (16)-(23) by operator splitting technique, we have obtained a sequence of simpler subproblems. To solve the "degenerated" quasi-Stokes problem (25), we employed a preconditioned conjugate gradient method introduced in [12] (see also [11] (Chapter 7)). In the above algorithm the preconditioning is achieved via the solution at each iteration of a discrete Poisson problem; to solve this last problem we used a matrix-free fast solver from FISHPAK, a package due to Adams, Swarztrauber and Sweet (see [1] for details). To solve the pure advection problem (26), we employed the wave-like equation method discussed in, e.g., [7], [11] (Chapter 6) and [21]. Unlike the two-dimensional flow investigated in [22], we have to solve here the two saddle-point problems (28) and (34). But when solving the saddle-point problem (34), the finite dimensional multiplier space $\Lambda_{0, h}^{n+s}$ has to verify some constraints. We have used conjugate gradient algorithms discussed in [23] to solve these two problems.

Systems (29)-(33) and (36)-(40) are systems of ordinary differential equations thanks to operator splitting. For its solution one can choose a time step smaller than $\Delta t$, (i.e., we can divide $\Delta t$ into smaller steps) to predict the translation velocity of the center of mass, the angular velocity of the particle, the position of the center of mass and the regions occupied by each particle so that the repulsion forces can be effective to prevent particle-particle and particle-wall overlapping. At each sub-cycling time step, keeping the distance constant between points $\mathbf{x}_{1}$ and $\mathbf{x}_{2}$ in each particle is important since we are dealing with rigid particles. To satisfy the above constraint we have applied the following approach:

- Translate $\mathbf{x}_{1}$ and $\mathbf{x}_{2}$ according to the new position of the mass center at each sub-cycling time step.

- Rotate $\mathbf{G x}_{1}$ and $\mathbf{G} \mathbf{x}_{2}$, the relative positions of $\mathbf{x}_{1}$ and $\mathbf{x}_{2}$ to the center of mass $\mathbf{G}$, by the following Crank-Nicolson scheme (a Runge-Kutta scheme of order 2, in fact):

$$
\frac{\mathbf{G x}_{i}^{\text {new }}-\mathbf{G x}_{i}^{\text {old }}}{\tau}=\omega \times \frac{\mathbf{G x}_{i}^{\text {new }}+\mathbf{G} \mathbf{x}_{i}^{\text {old }}}{2}
$$

for $i=1,2$ with $\tau$ as a sub-cycling time step. By (41), we have $\left|\mathbf{G x}_{i}^{\text {new }}\right|^{2}=\left|\mathbf{G} \mathbf{x}_{i}^{\text {old }}\right|^{2}$ for $i=1,2$ and $\mid \mathbf{G x}_{2}^{\text {new }}-$ $\left.\mathbf{G} \mathbf{x}_{1}^{\text {new }}\right|^{2}=\left|\mathbf{G} \mathbf{x}_{2}^{\text {old }}-\mathbf{G} \mathbf{x}_{1}^{\text {old }}\right|^{2}$ (i.e., scheme (41) is distance and in fact shape preserving).

Remark 7. In order to activate the short range repulsion force, we have to find the shortest distance between two ellipsoids. Unlike the cases for spheres, it is not trivial to locate the point from each surface of the ellipsoid where the distance is the shortest between two ellipsoids. There is no explicit formula for such distance. In practice, we first choose a set of points from the surface of each ellipsoid. 
Then we find the point among the chosen points from each surface at which the distance is the shortest. We repeat this (kind of relaxation) process in the neighborhood of the newly located point on each surface of ellipsoid until convergence, usually obtained in very few iterations.

For the shortest distance between the wall and ellipsoid, there exists an explicit formula. To check whether two ellipsoids overlap each other, there exists an algorithm used by people working on computer graphics and in robotics (e.g., see, [31]).

Remark 8. To get the angular velocity $\omega^{n+1}$, computed via $\boldsymbol{\omega}^{n+1}=\left(\mathbf{I}_{p}^{n+4 / 6}\right)^{-1}\left(\mathbf{I}_{p} \boldsymbol{\omega}\right)^{n+1}$,

we need to have $\mathbf{I}_{p}^{n+4 / 6}$, the inertia of the particle $B\left(t^{n+4 / 6}\right)$. We first compute the inertia $\mathbf{I}_{0}$ in the coordinate system attached to the particle. Then via the center of mass $\mathbf{G}^{n+4 / 6}$ and points $\mathbf{x}_{1}^{n+4 / 6}$ and $\mathbf{x}_{2}^{n+4 / 6}$, we have the rotation transformation $\mathbf{Q}\left(\mathbf{Q Q}^{\mathrm{T}}=\mathbf{Q}^{\mathrm{T}} \mathbf{Q}=\mathbf{I}_{d}, \operatorname{det} \mathbf{Q}=1\right)$ which transforms vectors expressed in the particle frame to vectors in the flow domain coordinate system and $\mathbf{I}_{p}^{n+s}=\mathbf{Q I}_{0} \mathbf{Q}^{\mathrm{T}}$. Actually in order to update matrix $\mathbf{Q}$ we can also use quaternion techniques, as shown, in the review paper [5].

\section{Numerical experiments and discussion}

\subsection{Rotation of a prolate spheroid}

For the first series of test problems, we have considered the simulation of a neutrally buoyant prolate ellipsoid moving in a fluid filled cylinder. Following Remark 5, we take for computational domain $\Omega=\left(0,1+4 h_{v}\right) \times(0,1+$ $\left.4 h_{v}\right) \times(0,2)$, with $h_{v}$ as the space discretization step to construct the flow velocity spaces. The radius $R$ of the cylinder is 0.5 and its length is 2 . The semi-long axis of the prolate ellipsoid is 0.195 and its two semi-short axes are 0.065 , while the common value of the densities of the fluid and particle is 1 . The viscosity of the fluid is either $0.5,0.1$, or 0.05 . The force $\mathbf{F}$ in (1) is a constant vector, positively oriented in the $O x_{3}$ direction; $\|\mathbf{F}\|$ has been chosen so that the maximum velocity of the corresponding Poiseuille flow (without particle) is 10. We suppose that the prolate ellipsoid is at rest initially and that the initial fluid velocity corresponds to the one of a fully developed Poiseuille flow of maximal velocity 10 . The mass center $\mathbf{G}(0)$ of the ellipsoid is vertically located below the cylinder axis at a distance 0.4 from this axis and the long axis of the ellipsoid lies on the plane parallel to the $x_{1} x_{3}$-coordinate plane. The initial angle between the long axis and the direction of the $x_{1}$-axis has been chosen as $0^{\circ}, 30^{\circ}$, or $90^{\circ}$. We have used uniform tetrahedral meshes to approximate velocity and pressure. The velocity (resp., pressure) mesh size is $h_{v}=1 / 80$ (resp., $h_{p}=2 h_{v}$ ), while the time discretization step is $\Delta t=0.001$.

For the cases of viscosity equal to 0.5 , the prolate ellipsoid has a stable orientational behavior after migrating away from the wall of the cylindrical tube and reaching its equilibrium distance to the central axis of the tube. Its long axis rotates on the plane passing through the cylinder axis and its mass center. This behavior is similar to the experimental results of the rod-like particle moving and rotating in the Poiseuille flow reported in [17]. The average distances of the mass center to the central axis of the tube are $0.5368 R, 0.5396 R$, and $0.5354 R$ for the initial angle of $0^{\circ}, 30^{\circ}$, and $90^{\circ}$, respectively, for $290 \leqslant t \leqslant 300$ as shown in Fig. 3. The center of mass moves along a straight line parallel to the $x_{3}$-axis after it has reached the equilibrium distance to the central axis of the cylindrical tube. The particle Reynolds numbers based on the length of the long axis and the average translation velocity for $290 \leqslant t \leqslant 300$ are $5.3807,5.3528$, and 5.3919 for the initial angle of $0^{\circ}, 30^{\circ}$, and $90^{\circ}$, respectively. Snapshots of the projection of the velocity field and the position of the prolate ellipsoid at $t=15.6,270,280,290$ for the case of initial angle of $0^{\circ}$ are shown in Figs. 4 and 5.

For the cases of viscosity equal to 0.1 , the prolate ellipsoid has two different rotational behaviors after reaching its equilibrium distance to the central axis of the tube. With the initial angle of $0^{\circ}$ and $30^{\circ}$, the prolate ellipsoid is rotating with respect to its long axis, which is perpendicular to the plane passing through the central axis of the tube and its mass center. This was not reported in Karnis, Goldsmith and Mason's 1964 paper [17] (see Figs. 6-8). The average distances of the mass center to the central axis of the tube for both initial angles of $0^{\circ}$ and $30^{\circ}$ are about $0.5192 R$ for $390 \leqslant t \leqslant 400$. Once the center of mass has reached the equilibrium distance to the central axis of the tube, it rotates with respect to the central axis of the tube as shown in Fig. 8. The particle Reynolds numbers based on the length of the long axis and the average translation velocity for $390 \leqslant t \leqslant 400$ are both about 26.23 . For the case of the initial angle equal to $90^{\circ}$, the prolate ellipsoid

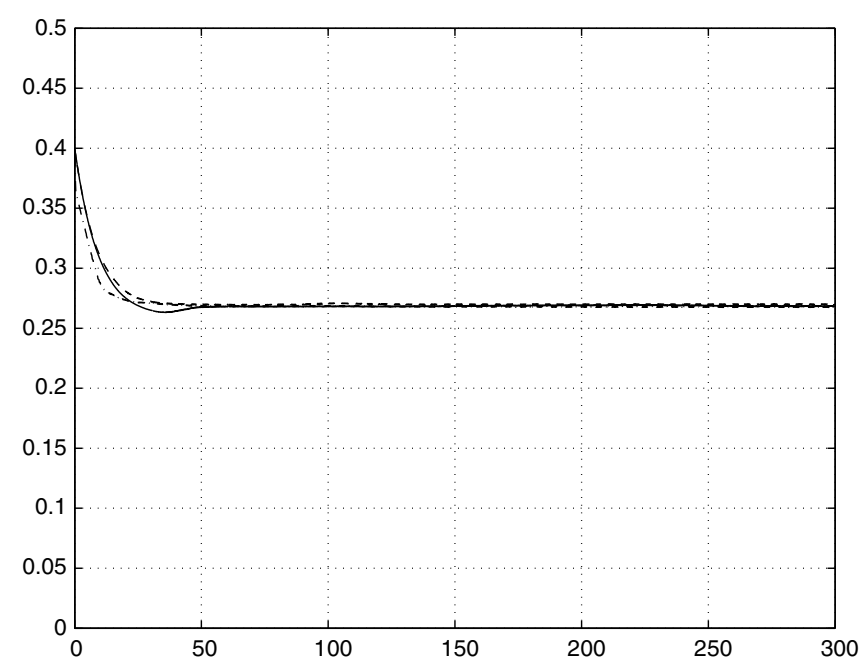

Fig. 3. Histories of the distance of the center of mass of the prolate ellipsoid from the central axis of the tube for the cases of initial angle of $0^{\circ}$ (solid line), $30^{\circ}$ (dashed line), and $90^{\circ}$ (dash-dotted line). 

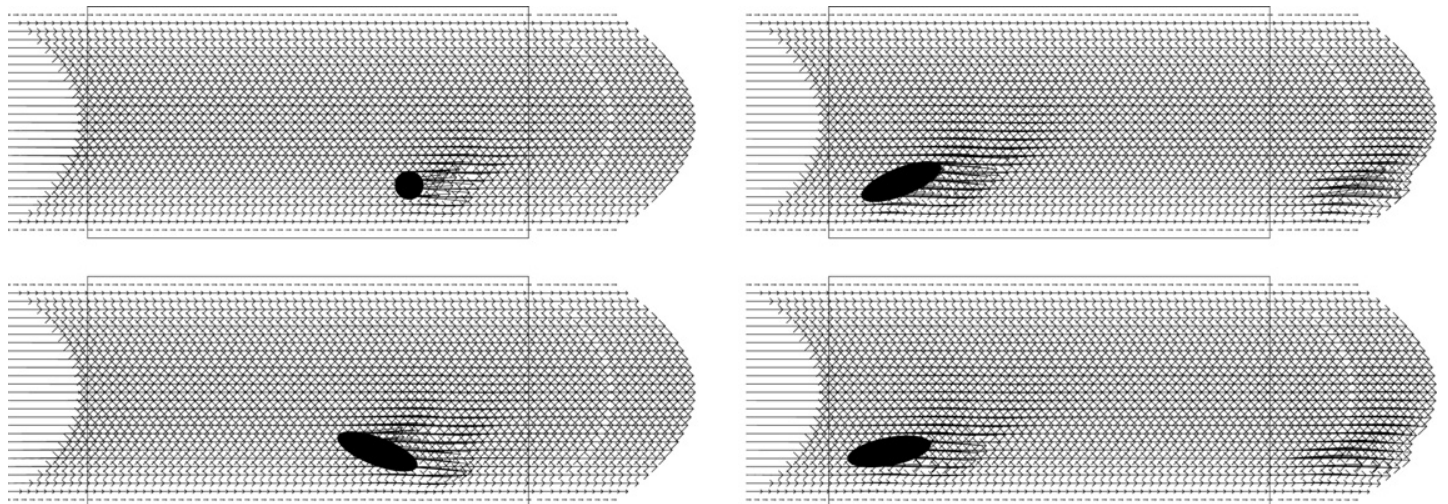

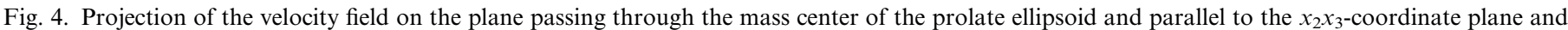

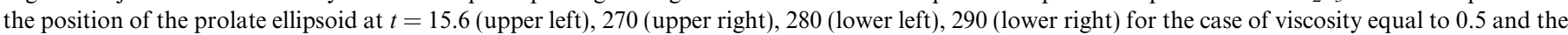
initial angle of $0^{\circ}$.
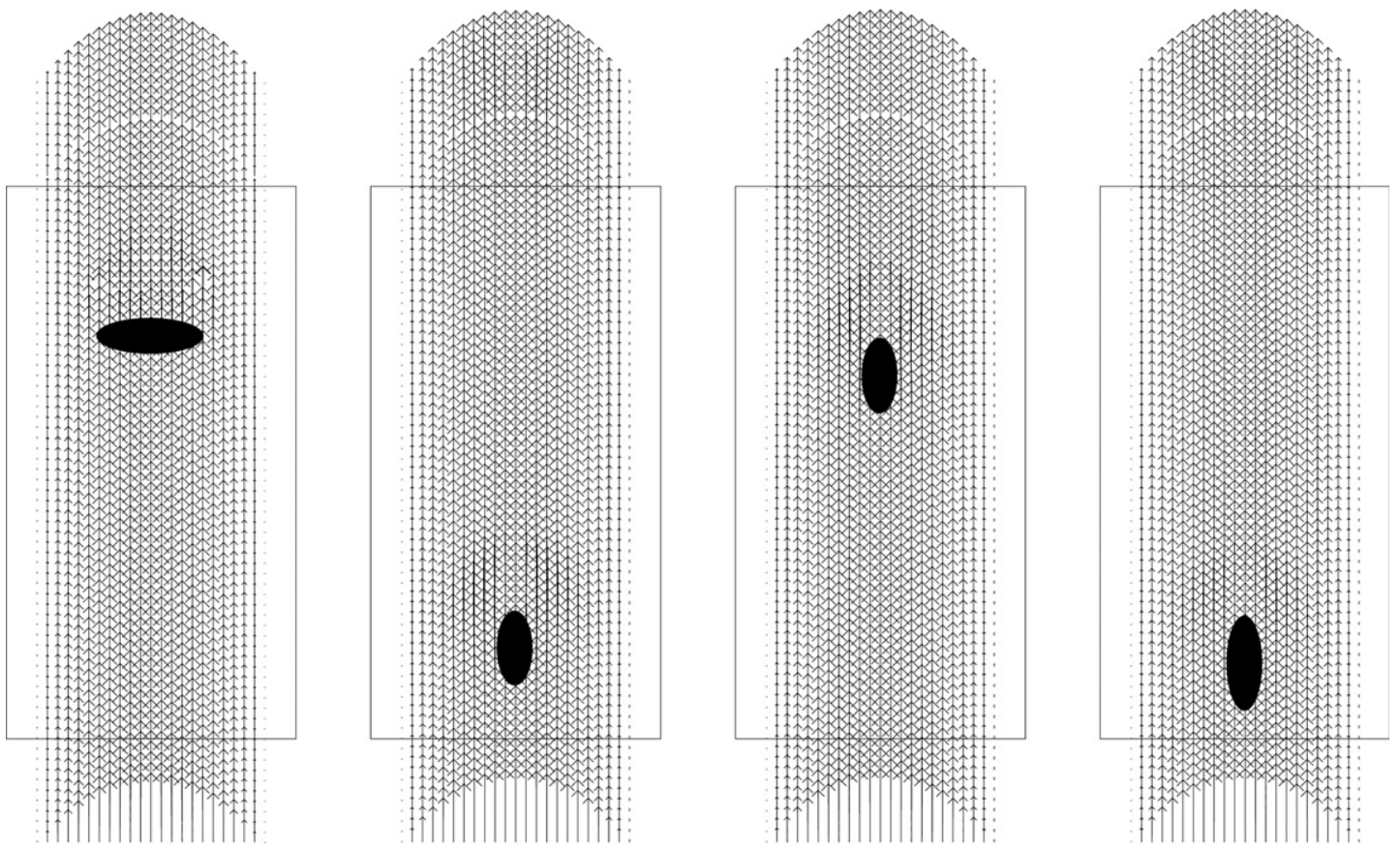

Fig. 5. Projection of the velocity field on the plane passing through the mass center of the prolate ellipsoid and parallel to the $x_{1} x_{3}$-coordinate plane and the position of the prolate ellipsoid at $t=15.6,270,280,290$ (from left to right) for the case of viscosity equal to 0.5 and the initial angle of $0^{\circ}$.

rotates with its long axis on the plane passing through the cylinder axis and its mass center just like it does for the case of viscosity equal to 0.5 . For $215 \leqslant t \leqslant 225$, the average distance of the mass center from the central axis of the tube is $0.5456 R$ and the particle Reynolds number based on the length of the long axis and the average translation velocity is 26.25 . We have obtained the same rotational behaviors when using a finer mesh size $h_{v}=1 / 120$ with time step $\Delta t=0.001$ and the initial angle between the long axis and the $x_{1}$-axis being $0^{\circ}$ and $90^{\circ}$; on the basis of this comparison we strongly believe that our results are not numerical artifacts.

For the cases of viscosity equal to 0.05 , the prolate ellipsoid again has two different rotational behaviors after reaching its equilibrium distance to the central axis of the tube as in the above case. When the initial angle is either $0^{\circ}, 30^{\circ}, 60^{\circ}$, or $90^{\circ}$, the prolate ellipsoid is rotating with respect to its long axis, which is perpendicular to the plane passing through the central axis and its mass center (see Fig. 9). The average distances of the mass center to the central axis of the tube are all about $0.5172 R$ and the particle Reynolds numbers based on the length of the long axis and the average translation velocity for $290 \leqslant t \leqslant 300$ are all about 52.36 for the four initial angles. When releasing the prolate ellipsoid at a position closer to the central axis (the distance is 0.252 instead of 0.4 ) with initial angle of $90^{\circ}$, the prolate ellipsoid rotates with its long axis on the plane passing through the cylinder axis and its mass center just like it does for the case of viscosity equal to 0.5 . The average distance of the mass center to the central axis of 

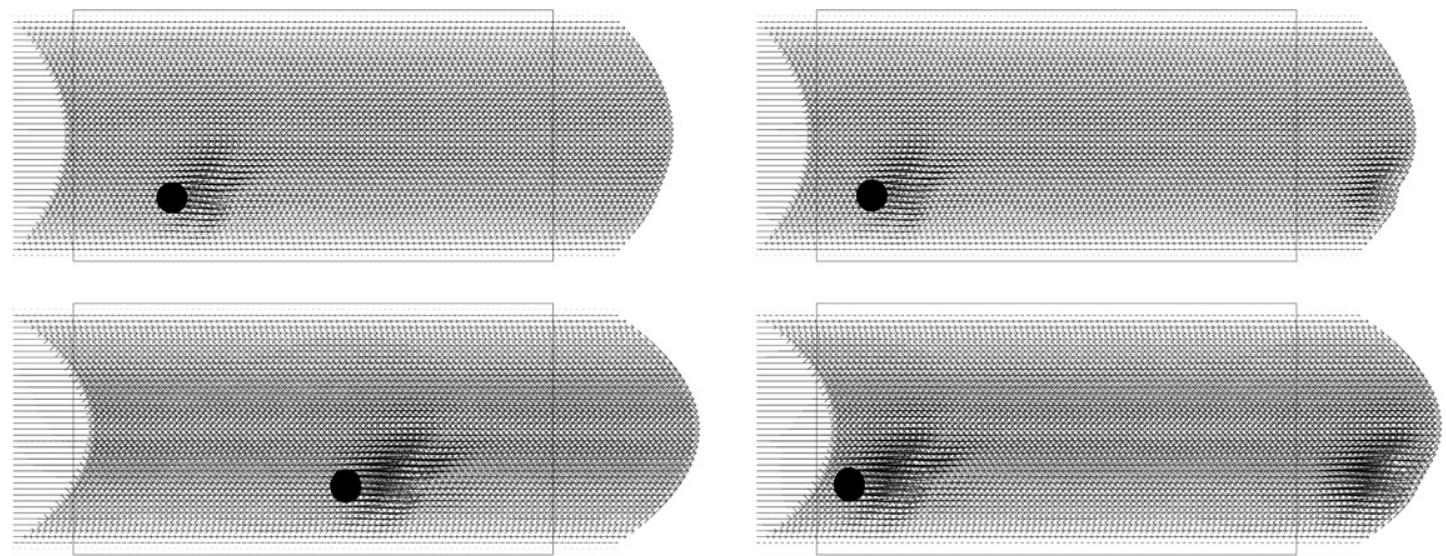

Fig. 6. Projection of the velocity field on the plane passing through the mass center of the prolate ellipsoid and parallel to the $x_{2} x_{3}$-coordinate plane and the position of the prolate ellipsoid at $t=15$ (upper left), 100 (upper right), 200 (lower left), 400 (lower right) for the case of viscosity equal to 0.1 and the initial angle of $0^{\circ}$.
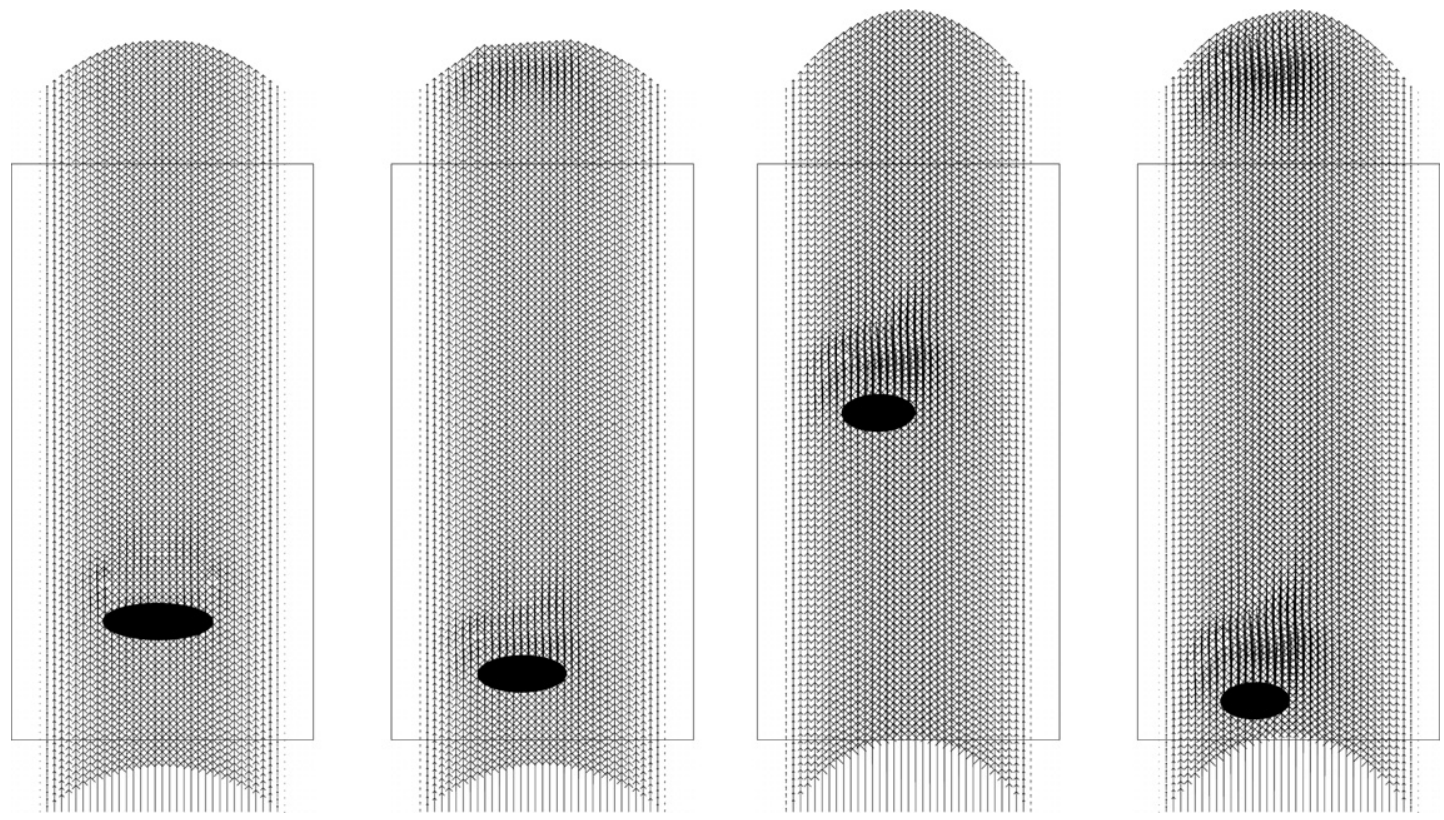

Fig. 7. Projection of the velocity field on the plane passing through the mass center of the prolate ellipsoid and parallel to the $x_{1} x_{3}$-coordinate plane and the position of the prolate ellipsoid at $t=15,100,200,400$ (from left to right) for the case of viscosity equal to 0.1 and the initial angle of $0^{\circ}$.
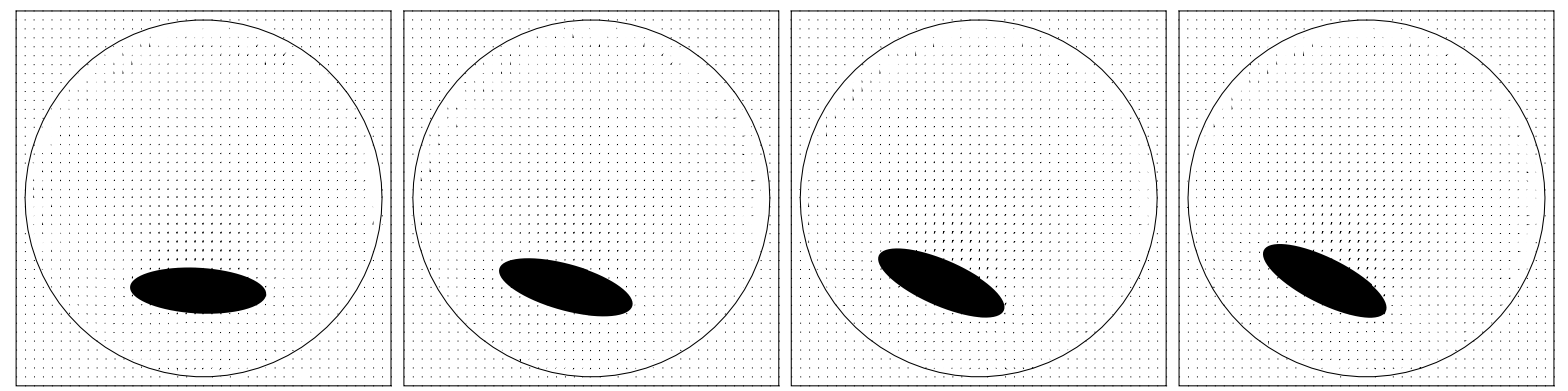

Fig. 8. Projection of the velocity field on the plane passing through the mass center of the prolate ellipsoid and parallel to the $x_{1} x_{2}$-coordinate plane and the position of the prolate ellipsoid at $t=15,100,200,400$ (from the left to the right) for the case of viscosity equal to 0.1 and the initial angle of $0^{\circ}$.

the tube is $0.5612 R$ and the particle Reynolds number based on the length of the long axis and the average trans- lation velocity of the mass center of the prolate ellipsoid is 50.92 for $215 \leqslant t \leqslant 225$. 

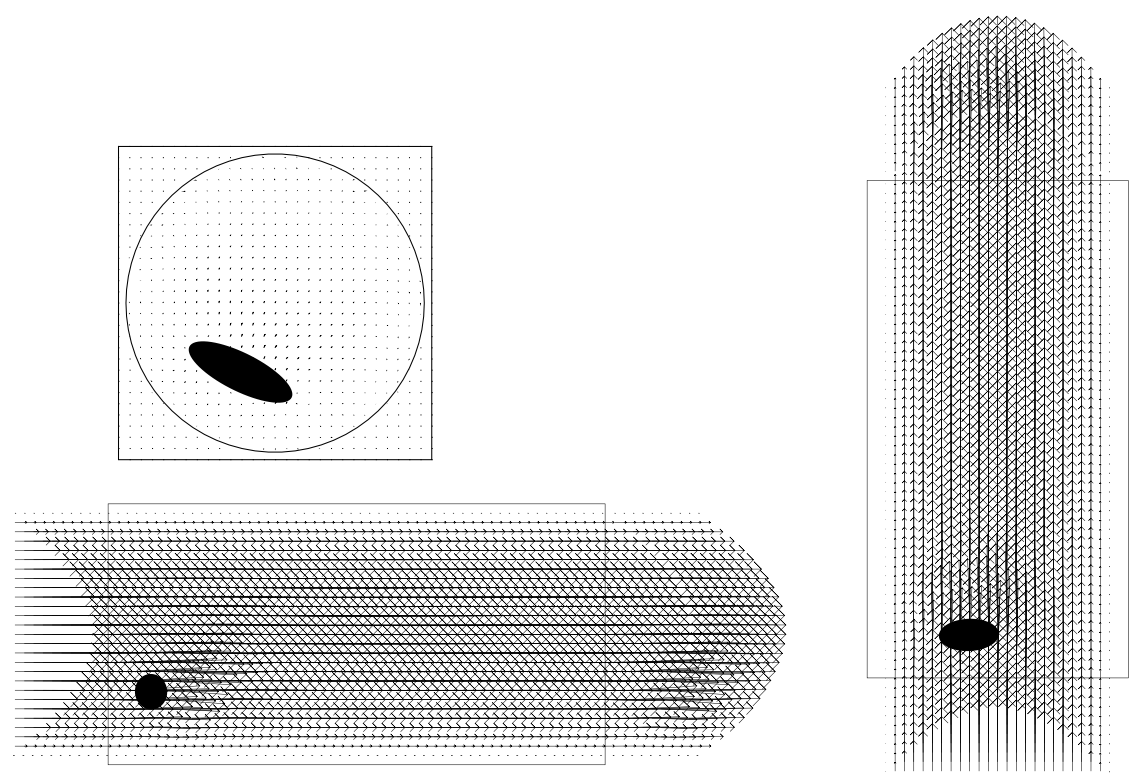

Fig. 9. Projection of the velocity field on the plane passing through the mass center of the prolate ellipsoid and parallel to the $x_{1} x_{2}$-coordinate plane (upper left), the $x_{2} x_{3}$-coordinate plane (lower left), the $x_{1} x_{3}$-coordinate plane (right) at $t=300$ for the case of viscosity equal to 0.05 and the initial angle of $90^{\circ}$.

In this section, for a neutrally buoyant prolate ellipsoid moving in a three-dimensional tube Poiseuille flow, we have obtained two different rotational states and one of them was not reported in Karnis, Goldsmith and Mason's 1964 paper [17]. Qi and Luo also obtained similar results in $[29,30]$. But the difference is that we have obtained the coexistence of two rotational behaviors at the same range of the particle Reynolds number and Qi and Luo did not obtain it. We believe that the flow field is strong enough to stabilize the rotation with respect to the long axis of the ellipsoid when the viscosities are 0.1 and 0.05 . Also the symmetric cross-section of the tube might also play a role here since in $[29,30]$, Qi and Luo considered Couette flow, which does not have such symmetric property. The ratio of the length of the long axis and the radius of the tube and the initial position of the prolate might be other important factors for stabilizing the orientation of the prolate ellipsoid. We will investigate and report the effect of the viscosity and the length of long axis of the prolate ellipsoid in the near future.

\subsection{Rotation of an oblate spheroid}

For the second series of test problems, we have considered the simulation of a neutrally buoyant oblate ellipsoid moving in a fluid filled cylinder. We have chosen a set of
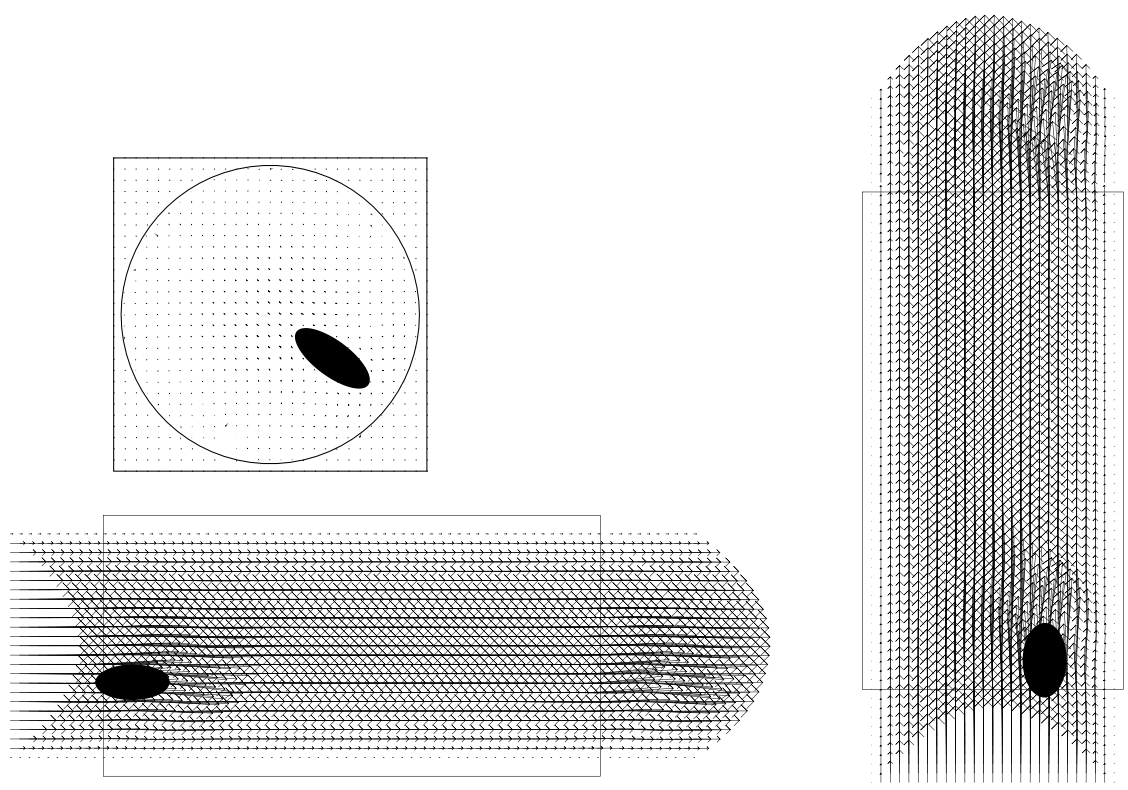

Fig. 10. Projection of the velocity field on the plane passing through the mass center of the oblate ellipsoid and parallel to the $x_{1} x_{2}$-coordinate plane (upper left), the $x_{2} x_{3}$-coordinate plane (lower left), the $x_{1} x_{3}$-coordinate plane (right) at $t=295$ for the case of viscosity 0.5 . 

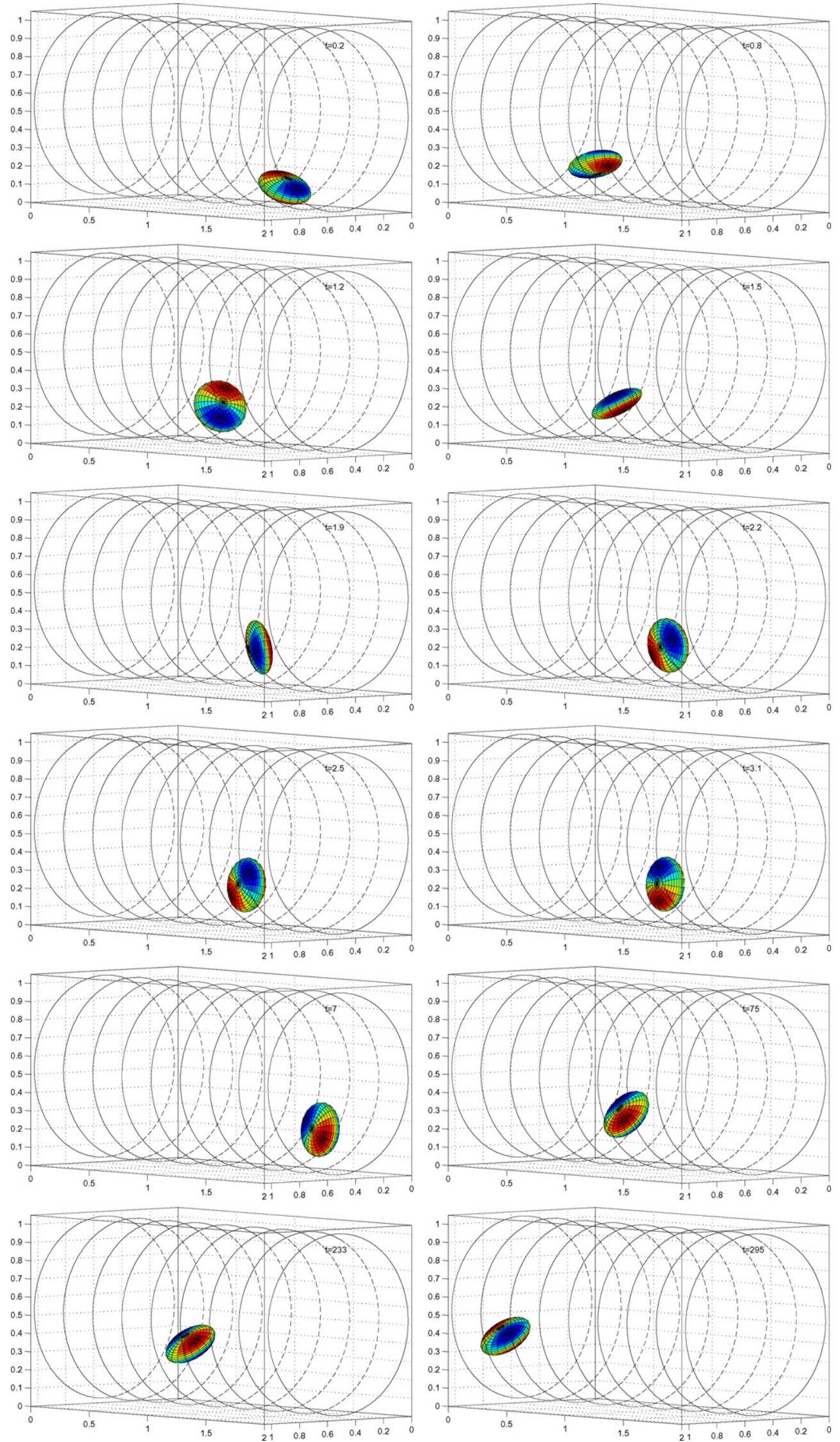

Fig. 11. Position of the oblate ellipsoid at $t=0.2,0.8,1.2,1.5,1.9,2.2,2.5,3.1,7,75,233$, and 295 (from left to right and from top to bottom) for the case of viscosity 0.5 . 
parameters and initial conditions almost identical as the one in the above series of test problems; the difference are: (i) the two semi-long axes are 0.15 and the semi-short axis is 0.06 , (ii) the mass center is initially placed under the central axis of the tube so that the plane parallel to the $x_{1} x_{3}$-coordinate plane contains two long axes and the distance from the center of mass to the central axis of the tube is 0.415 , and (iii) the viscosity of the fluid is either $0.5,0.1$, 0.05 or 0.025 . For all four different viscosities considered in this section, the oblate ellipsoid has a stable orientational behavior after migrating away from the wall of the cylindrical tube and reaching its equilibrium distance to the central axis of the tube (see Figs. 10 and 11), which is that an oblate ellipsoid rotates with its short axis perpendicular to the plane passing through the central axis of the tube and the mass center of the disk. The average distances of the mass center to the central axis of the tube are $0.507 R$, $0.5102 R, 0.521 R$, and $0.5186 R$ for the viscosity $0.5,0.1$, 0.05 , and 0.025 , respectively, for $170 \leqslant t \leqslant 180$. The particle Reynolds numbers based on the length of the long axis and the average translation velocity for $170 \leqslant t \leqslant 180$ are $4.226,20.55,40.38$, and 81.28 for the viscosity $0.5,0.1$, 0.05 , and 0.025 , respectively. This behavior is similar to the experimental results for the disk-like particle moving and rotating in the Poiseuille flow reported in [17].

\section{Conclusion}

In this article, we have discussed the application of a distributed Lagrange multiplier fictitious domain method for simulating the motion of neutrally buoyant ellipsoids in a three-dimensional tube Poiseuille flow and applied this methodology to study their rotational behavior in such flow. We found distinctive states depending on the Reynolds number ranges and the shape of the ellipsoids. We will investigate the cases with different sizes of ellipsoid and wider range of viscosity to find out their effects on the motion of a neutrally buoyant ellipsoid in a threedimensional tube Poiseuille flow.

\section{Acknowledgements}

We acknowledge the helpful comments and suggestions of E.J. Dean, J.W. He, H.H. Hu, G.P. Galdi, P. Huang, D.D. Joseph, Y. Kuznetsov, V. Paulsen, and G. Rodin. The support of NSF (grants ECS-9527123, CTS-9873236, DMS-9973318, CCR-9902035, DMS-0209066, and DMS0443826) and DOE/LASCI (grant R71700K-292-000-99) is also acknowledged.

\section{References}

[1] J. Adams, P. Swarztrauber, R. Sweet, FISHPAK: A Package of Fortran Subprograms for the Solution of Separable Elliptic Partial Differential Equations, The National Center for Atmospheric Research, Boulder, CO, 1980.
[2] T. Bertrand, P.A. Tanguy, F. Thibault, A three-dimensional fictitious domain method for incompressible fluid flow problems, Int. J. Numer. Methods Fluids 25 (1997) 719-736.

[3] H. Brenner, Hydrodynamic resistance of particles at small Reynolds numbers, Adv. Chem. Engrg. 6 (1966) 287-438.

[4] M.O. Bristeau, R. Glowinski, J. Periaux, Numerical methods for the Navier-Stokes equations. Applications to the simulation of compressible and incompressible viscous flow, Comput. Phys. Rep. 6 (1987) 73-187.

[5] J.C.K. Chou, Quaternion kinematic and dynamic differential equations, IEEE Trans. Robotic Autom. 8 (1992) 53-64.

[6] R.G. Cox, S.G. Mason, Suspended particles in fluid flow through tubes, Ann. Rev. Fluid Mech. 3 (1971) 291-316.

[7] E.J. Dean, R. Glowinski, A wave equation approach to the numerical solution of the Navier-Stokes equations for incompressible viscous flow, CR Acad. Sci. Paris, Série 1325 (1997) 783-791.

[8] E.J. Ding, C.K. Aidun, The dynamics and scaling law for particles suspended in shear flow with inertia, J. Fluid Mech. 423 (2000) $317-$ 344.

[9] J. Feng, H.H. Hu, D.D. Joseph, Direct simulation of initial value problems for the motion of solid bodies in a Newtonian fluid. Part 2: Couette and Poiseuille flows, J. Fluid Mech. 277 (1994) 271-301.

[10] F. Feuillebois, Some theoretical results for the motion of solid spherical particles in a viscous fluid, in: G.F. Hewitt, J.M. Delhaye, N. Zuber (Eds.), Multiphase Science and Technology, vol. 4, Hemisphere Publishing Corporation, New York, 1989, pp. 583-798.

[11] R. Glowinski, Finite element methods for incompressible viscous flow, in: P.G. Ciarlet, J.L. Lions (Eds.), Handbook of Numerical Analysis, vol. IX, North-Holland, Amsterdam, 2003, pp. 3-1176.

[12] R. Glowinski, T.-W. Pan, J. Periaux, Distributed Lagrange multiplier methods for incompressible flow around moving rigid bodies, Comput. Method Appl. Mech. Engrg. 151 (1998) 181-194.

[13] R. Glowinski, T.-W. Pan, T. Hesla, D.D. Joseph, A distributed Lagrange multiplier/fictitious domain method for particulate flows, Int. J. Multiphase Flow 25 (1999) 755-794.

[14] R. Glowinski, T.-W. Pan, T. Hesla, D.D. Joseph, J. Periaux, A fictitious domain approach to the direct numerical simulation of incompressible viscous flow past moving rigid bodies: application to particulate flow, J. Comput. Phys. 169 (2001) 363-426.

[15] E.Y. Harper, I.-D. Chang, Maximum dissipation resulting from lift in a slow viscous shear flow, J. Fluid Mech. 33 (1968) 83-102.

[16] G.B. Jeffery, The motion of ellipsoidal particles immersed in a viscous fluid, Proc. Roy. Soc. London, Ser. A. 102 (1922) 161-179.

[17] A. Karnis, H.L. Goldsmith, S.G. Mason, The flow of suspensions through tubes. Part V: inertial effects, Can. J. Chem. Engrg. 44 (1966) 181-193.

[18] L.G. Leal, Particle motions in a viscous fluid, Ann. Rev. Fluid Mech. 12 (1980) 435-476.

[19] G.I. Marchuk, Splitting and Alternating Direction Methods, in: P.G. Ciarlet, J.L. Lions (Eds.), Handbook of Numerical Analysis, vol. I, North-Holland, Amsterdam, 1990, pp. 197-462.

[20] J.B. McLaughlin, Inertial migration of a small sphere in linear shear flows, J. Fluid Mech. 224 (1991) 261-274.

[21] T.-W. Pan, R. Glowinski, A projection/wave-like equation method for the numerical simulation of incompressible viscous fluid flow modeled by the Navier-Stokes equations, Comput. Fluid Dynam. J. 9 (2000) 28-42.

[22] T.-W. Pan, R. Glowinski, Direct simulation of the motion of neutrally buoyant circular cylinders in plane Poiseuille flow, J. Comput. Phys. 181 (2001) 260-279.

[23] T.-W. Pan, R. Glowinski, Direct simulation of the motion of neutrally buoyant balls in a three-dimensional Poiseuille flow, CR Mecanique 333 (2005) 884-895.

[24] T.-W. Pan, R. Glowinski, D.D. Joseph, Simulating the dynamics of fluid-cylinder interactions, J. Zhejiang Univ. Sci. 6A (2005) 97-109.

[25] T.-W. Pan, R. Glowinski, D.D. Joseph, Simulating the dynamics of fluid-ellipsoid interactions, Comput. Struct. 83 (2005) 463-478. 
[26] C.S. Peskin, Numerical analysis of blood flow in the heart, J. Comp. Phys. 25 (1977) 220-252.

[27] C.S. Peskin, D.M. McQueen, Modeling prosthetic heart valves for numerical analysis of blood flow in the heart, J. Comp. Phys. 37 (1980) 113-132.

[28] C.S. Peskin, Lectures on mathematical aspects of physiology, Lectures Appl. Math. 19 (1981) 69-107.

[29] D.W. Qi, L.S. Luo, Transitions in rotations of a non-spherical particle in a three-dimensional moderate Reynolds number Couette flow, Phys. Fluid 14 (2002) 4440-4443.

[30] D.W. Qi, L.S. Luo, Rotational and orientational behavior of threedimensional spheroidal particles in Couette flow, J. Fluid Mech. 477 (2003) 201-213.
[31] E. Rimon, S. Boyd, Efficient distance computation using best ellipsoid fit.t, Technical report, Information Systems Laboratory, Stanford University, 1992.

[32] G. Segré, A. Silberberg, Radial particle displacements in Poiseuille flow of suspensions, Nature 189 (1961) 209-210.

[33] G. Segré, A. Silberberg, Behavior of macroscopic rigid spheres in Poiseuille flow. Part I, J. Fluid Mech. 14 (1962) 115-157.

[34] B.H. Yang, J. Wang, D.D. Joseph, H.H. Hu, T.-W. Pan, R. Glowinski, Migration of a sphere in tube flow, J. Fluid Mech. 540 (2005) 109-131.

[35] Z. Yu, N. Phan-Thien, R.I. Tanner, Dynamic simulation of sphere motion in a vertical tube, J. Fluid Mech. 518 (2004) 61-93. 